\title{
Event-based low-frequency impedance modeling using well logs and seismic attributes
}

\author{
Radmila Pedersen-Tatalovic, Anette Uldall, and Nils Lange Jacobsen, Maersk Oil, Copenhagen, Denmark \\ Thomas Mejer Hansen and Klaus MosegaARD, the Niels Bohr Institute, Copenhagen, Denmark
}

\begin{abstract}
A coustic impedance is a rock property that, under specific conditions, can be derived from seismic data and can provide important insights into reservoir parameters—such as porosity, lithology, fluid content, etc. Direct measurements of acoustic impedance are available from sonic and density well logs. Seismic inversion, a process of converting seismic data into relative impedance, provides estimates of relative acoustic impedance away from the well locations. Because absolute acoustic impedance can be related to other rock properties, the inverted relative seismic impedance could be used to predict these properties away from the wells if the missing low frequencies could be reliably calculated and compensated for. In chalk, seismic inversion finds its most significant application in porosity prediction.
\end{abstract}

Compared to the well acoustic impedance, inverted relative acoustic impedance has a limited bandwidth, which is restricted on low and high ends of the frequency spectrum. Band-limited impedance (in this article referred to as relative impedance) is a useful seismic attribute for a better qualitative understanding of reservoir properties, and it often can be used for quantitative estimation of other reservoir properties, especially in clastic reservoirs. Since chalk consists of homogeneous lithofacies with clean matrix character with its porosity as the main variable, it is a perfect medium for qualitative prediction of this specific attribute. However, quantitative reservoir characterization in chalk is severely limited by the lack of low-frequency information because the bulk of the strong correlation between impedance and porosity is carried by the low-frequency trend.

The missing low-frequency part of the inverted impedance data can be modeled by lateral interpolation of impedance logs between well locations. Conventionally, this interpolation has been driven by distance between the wells, which often leads to artifacts and generation of nongeologic solutions. Distance-based, well-log interpolation can be significantly improved by using seismic velocities to guide the well-log interpolation. Although the use of velocities is a major improvement, it is limited by seismic resolution and accuracy that generally deteriorates with depth. Velocity data only partially provide the missing information in the lowest frequency range.

In the present study, event-based validated multivariate interpolation was successfully applied to recover lowfrequency acoustic impedance using well data, velocity data, and seismic attributes. The examples given in this article originate from the Danish sector in the North Sea. Using seismic estimates of interval velocities, layer depths, formation thicknesses, and reflection amplitudes, it was possible to significantly improve the accuracy of the predicted low-frequency response and therefore the porosity estimates as compared to conventional methods. Impedance information in the frequency interval between 0 and somewhat higher than $8 \mathrm{~Hz}$ was estimated, achieving an improvement especially between 2 and $8 \mathrm{~Hz}$, where neither seismic velocities (with the bandwidth of approximately $0-2 \mathrm{~Hz}$ ) nor seismic

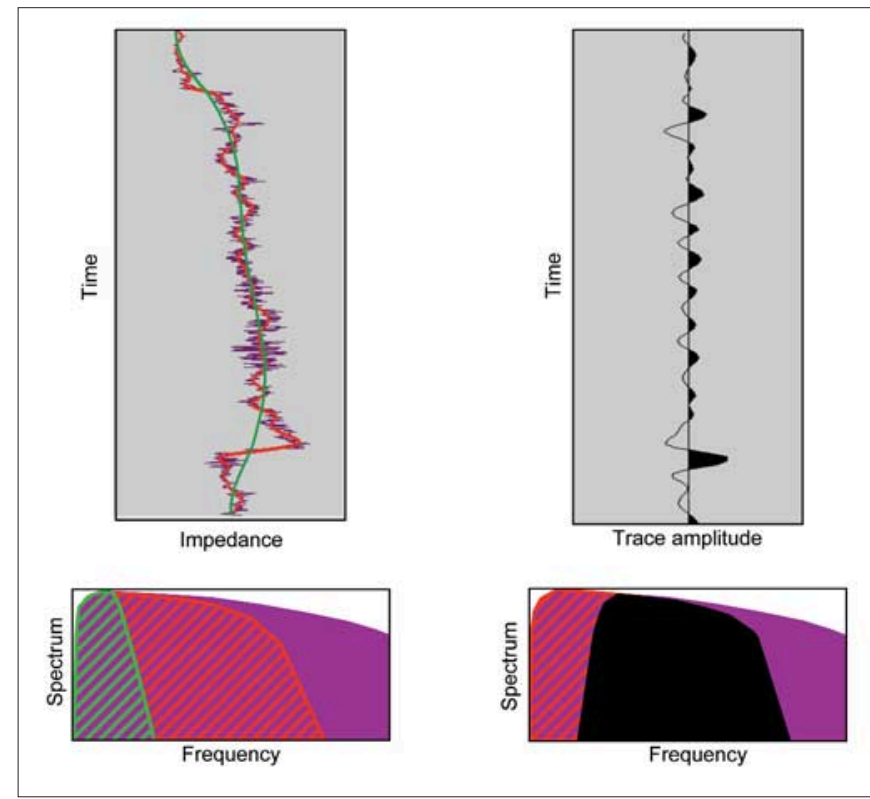

Figure 1. (left) Well log (upper panel) and the corresponding frequency spectrum, in original frequency range (purple), high-cut filtered to $60 \mathrm{~Hz}$ (red), and $8 \mathrm{~Hz}$ (green). (right) Seismic trace (upper panel) and its frequency range (lower panel) in black.

data (with the bandwidth of approximately $8 \mathrm{~Hz}$ and higher) provide adequate information.

Introduction and historical background. Seismic inversion is a process that converts seismic trace information into acoustic impedance. Through the inverted impedance, other reservoir properties-such as lithology, porosity, and fluid content-can be quantified away from the wells.

Well logs are normally measured at .5-ft resolution and therefore have a broad spatial frequency response that in the time domain corresponds to a range from close to zero to several thousands of $\mathrm{Hz}$. Conventional seismic reflection data are band-limited to $125 \mathrm{~Hz}$ with the typical sample rate of $4 \mathrm{~ms}$, although the useful signal bandwidth usually does not exceed the range of approximately $6-60 \mathrm{~Hz}$. The practical band limitation is a consequence of the geometry of the recording layout, the sample rate, source pulse (its energy and frequency), and the elastic response and dampening of the subsurface. Compared to the well logs, seismic data have limited bandwidth on low and high ends. An example of an impedance well log and its corresponding spectrum is displayed in purple (left) on Figure 1. Note how resolution decreases when the same well log and its spectrum are high-cut filtered to the upper limit of the seismic frequency range (at about $60 \mathrm{~Hz}$ ), displayed in red. The high end of this spectrum compares with that of the seismic trace, shown in black on the right panel. Just as the well logs have lower resolution because of the lack of high fre- 
quencies (larger than about $60 \mathrm{~Hz}$ ), so do the seismic data and inverted impedance. Nevertheless, the curve displayed in red could be considered an ideal result from seismic inversion.

Well-log impedance shows a trend, displayed in green in Figure 1, of generally increasing values with depth. Seismic data do not by definition have any inherent trend with time- the positive and negative amplitude deviations along the trace even out-because seismic samples do not represent any frequencies lower than approximately the trend shown in green. It is this low-frequency trend that is essential to properly recover when inverting seismic data. At well locations this is easily done by calculating acoustic impedance from sonic and density well logs. Away from the well, the low-frequency information is modeled by interpolating the well logs.

Traditionally, missing high frequency in inverted seismic data has been considered more limiting than missing low frequency. Consequently, various inversion methods have been proposed to retrieve the high frequencies mainly for improved resolution. Although not disputing the importance of the high frequency, we demonstrate the importance of the low frequency and propose a method for recovering the missing low frequencies in order to produce a more accurate reservoir characterization.

Figure 2 shows impedance logs from two wells in the same region. On each panel, the impedance log and its lowfrequency component are shown to the right, and the corresponding relative impedance is shown to the left. The relative impedance is created by filtering out the low-frequency component from the well log. For the well log shown on the left panel, the relative impedance log shows a large deviation at about $2 \mathrm{~s}$, and some increased reflectivity around 1.6-1.8 s. It is not possible to detect the slow build-up of absolute impedance values on the relative impedance log, in the relatively thick section between 1.6 and 2 s. Similarly, on the right panel, the relative impedance shows an increased reflectivity between 1.9 and $2.1 \mathrm{~s}$, but does not reveal the relatively quick build-up of high absolute impedance and then relatively quick decrease of absolute values. It is clear from these displays that for both wells the lowfrequency component is essential in recognizing the absolute level of impedance. The inverted impedance containing the replaced low-frequency component is defined as absolute impedance.

Importance of low-frequency information. In spite of its limitations, relative impedance can be a very useful attribute, because its higher resolution can be used for detailed stratigraphic interpretation and it often provides additional information about essential reservoir properties. Relative impedance can be used as a lithology, porosity, or hydrocarbon indicator, especially in sand-shale sequences, as long as a relationship between the relative impedance and the other reservoir properties exists and can be established through well-log crossplotting at the appropriate band-limited frequencies.

In clean chalk, impedance can optimally be used to predict porosity, because it has a strong correlation to porosity, and because its sensitivity to the presence of hydrocarbon fluids is limited. The presence of free gas or a high level of solution gas in the reservoir is normally handled by correcting the impedance level accordingly. Consequently, in order to derive porosity from impedance in chalk, it is essential to recover the low frequencies and establish the absolute impedance level. This is illustrated in Figure 3, which shows a crossplot of impedance and porosity logs within the chalk

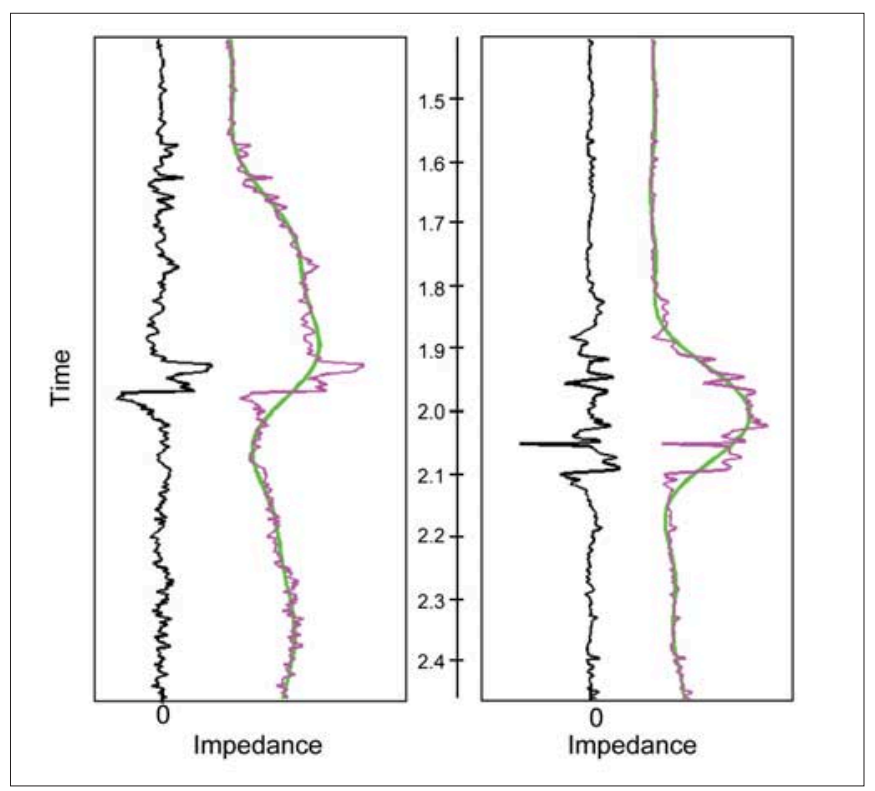

Figure 2. Impedance log (purple) and its low-frequency component (green). Relative impedance (black), is created by subtracting the lowfrequency component from the impedance log. The purple curve is also referred to as absolute impedance.

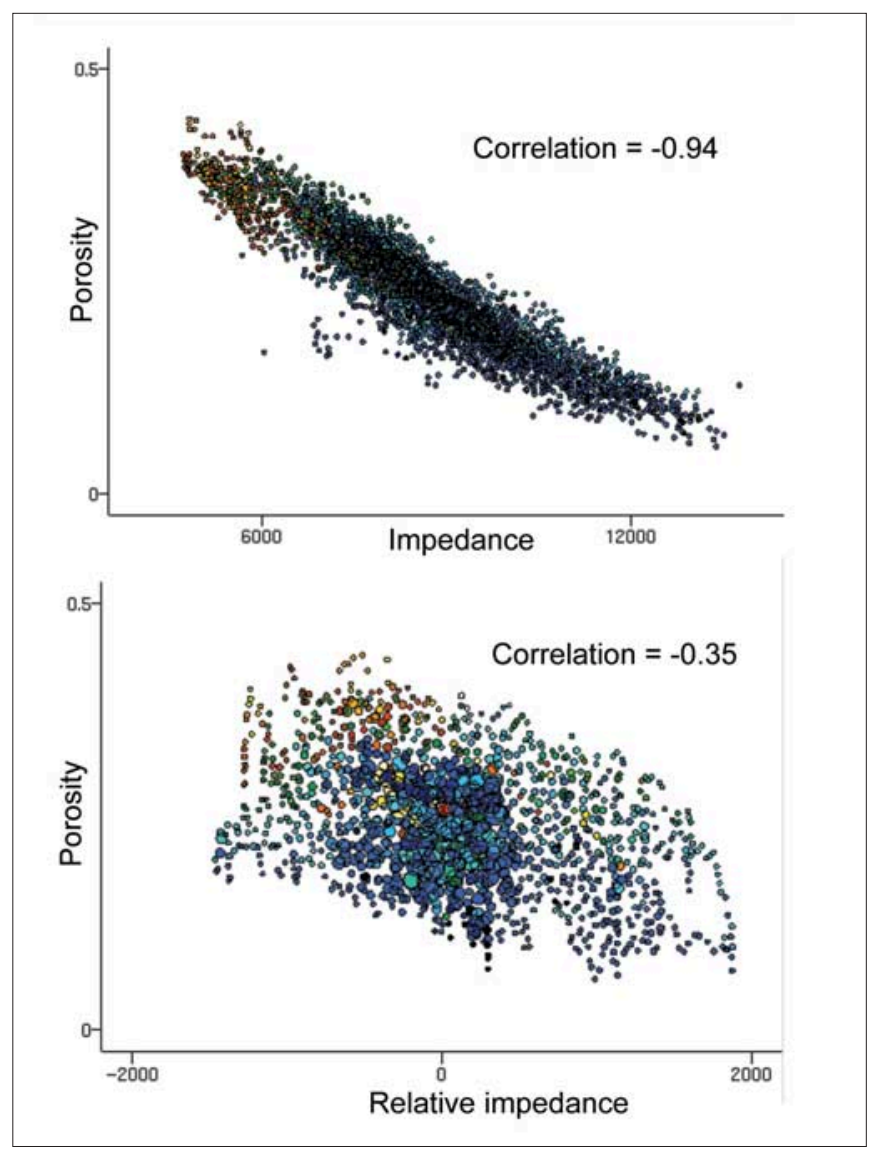

Figure 3. Crossplot of chalk impedance and porosity logs for a number of wells in the Danish sector of the North Sea. Absolute impedance on the upper panel, relative impedance on the lower panel. Blue represents high water saturation, yellow represents low water saturation.

formation for a number of wells from the Danish sector, color-coded with water saturation; warm colors indicate low water saturation. The upper plot was generated from the full-bandwidth impedance logs, and the lower plot was 

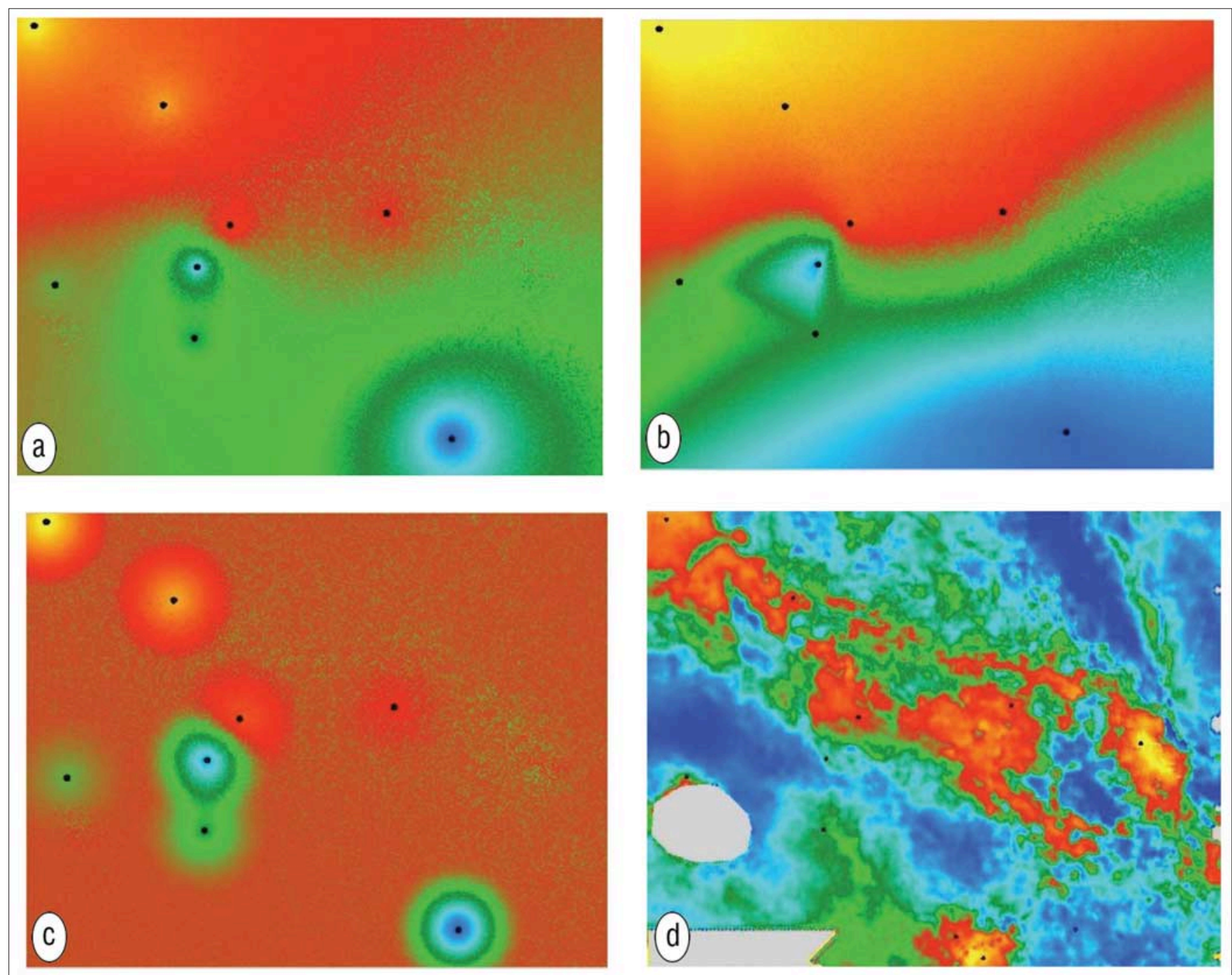

Figure 4. $(a, b$, and c) Distance-based interpolation of well logs with various attempts to capture regional trend variation; (d) attribute-driven interpolation of well logs derived from this study. The eastern-most well and the two southern-most wells were not used in the distance-based interpolation.

generated from band-pass filtered logs with filter settings adjusted to approximate seismic bandwidth. Note the superior correlation in the upper plot. Observe also that the low water saturation is associated only with low absolute impedance values, clearly indicating a hydrocarbon effect. The hydrocarbon effect cannot be recognized on the lower plot showing the relative impedance-to-porosity correlation. Therefore, in order to use inverted impedance for porosity estimation in chalk, it is essential to include the full-frequency spectrum, and the seismic frequencies need to be correctly compensated for within the missing low part of the spectrum.

Recovering low-frequency impedance with existing methods. The low-frequency data missing in the inverted impedance are usually generated via simplified Earth models, which are typically built by interpolating well logs guided by a gross-layer model containing only a few interpreted seismic horizons. Traditional methods usually employ some sort of mathematical interpolation routine, guided primarily by the distance between the wells and using the interpreted seismic horizons to link the individual well logs. By the very nature of the method, this technique usually fails to capture any lateral change of geologic information or trends. An extreme example is given in Figures $4 a-c$, that illustrates results from three different distance-based interpolation methods. The well locations are shown in black, and impedance (in color) has been averaged over a zone of interest. The first display (Figure 4a) shows the low-frequency component that results from an inverse distance method, where at each point the influence of any well is calculated as an inverse square of its distance. The next two displays ( $4 \mathrm{~b}$ and c) use a similar approach with an additional attempt to create a more regional model and take into account the distribution, density, and mean of the data points. All displays illustrate the risk of misrepresenting the geology in case of sparsely sampled well data when interpolation is driven by distance only. These results should be compared with the last display (Figure 4d) that results from using an interpolation method that recovers the low-frequency component not only by strict mathematical interpolation of the well data points, but also takes geologic information into account. A completely distance-based technique not only fails to capture lateral variations in geologic trends, but it also fails to include that very low-frequency information recoverable from seismic velocities.

A number of methods are described in the literature that allow well interpolation to be guided by external attributes such as linear regression, kriging and cokriging, and neural networks. Neural network methods can handle strongly 
nonlinear problems, and they can work with many types of input data. Liu and Liu (1998) show how a multilayer feedforward neural network can be used, and Hampson et al. (2001) illustrate a use of a probabilistic neural network to predict log properties. See Herrera et al. (2006) for a recent application of a neural network for the estimation of borehole log properties using seismic attributes. The method we propose can be compared to that of a linear neural network, although our choice of parameterization allows the method to give stable predictions using relatively few data points.

Cokriging can be used to interpolate well logs using a set of attributes and a covariance model derived from the data. To derive an appropriate covariance model, which is essential for successful application, a large number of data points are required; however, in most practical applications, use of more than one attribute is not considered feasible. Furthermore, kriging techniques rely on the fundamental assumption that proximate data points are more correlated than the more distal data points, which is not always the case. A comparison of cokriging and neural networks-based techniques can be found in Trappe and Hellmich (2000).

The method utilized in this study does not assume that spatially close data are necessarily more correlated, and it requires relatively few input data points for stable performance. Furthermore, the method estimates a prediction operator that is easy to analyze and provides reliable uncertainty estimates for all predictions.

The current method resulted from experience gained during a regional inversion project of $3 \mathrm{D}$ seismic data from the Central Graben area of the Danish North Sea. In this project, a major effort was undertaken to improve the conventional method of well interpolation for compensating the low-frequency information. A more-or-less manual method was applied to control the well-log interpolation not only by distance, but also to guide the interpolation by seismic velocity and thickness of interpreted chalk facies.

Although superior results were achieved compared to the available standard results, it was a rather cumbersome procedure because of the lack of an automated process. Furthermore, it was recognized that the method had to be further refined and generalized to allow use of multiple attributes to control the significance of each individual attribute or a combination of attributes, and to extend and balance the required full-frequency bandwidth.

Based on this experience, the current method was developed, including a software tool for easy application. The calculation module allows multiple attributes and controls their relationship with the well logs. The method applies a geologic similarity criterion: If the geology at two locations, determined by a range of attributes, is deemed similar, then the well logs at these locations are expected to be similar. The tool is not designed to provide a full inversion but aims at filling the missing low frequencies-not only because satisfactory band-limited inversion software exists on the market, but also because focusing specifically on low frequencies allows a relatively sparse vertical parameterization.

Event-based validated multivariate interpolation. The basic challenge can be formulated as follows: How can a relationship be determined between subsurface seismic data in an area and a known set of corresponding subsurface acoustic-impedance well-log data points? The basic idea is to derive a more general relationship between seismic attributes and low-frequency acoustic impedance values analyzing the correlation between these parameters when observed at a limited number of well locations. The derived transfer function can be subsequently used to predict the low-fre- quency impedance response anywhere in the region based solely on the seismic attribute response. Impedance measurements are available from a number of well logs that represent much sparser data coverage compared to that of the available seismic attributes. The seismic information is expressed in the form of seismic attributes, which are selected based on their ability to describe the underlying geology. This is not a specific requirement of the method because the initial data analysis will automatically determine the importance of the attribute included in the prediction process. For example, the attributes utilized could vary: reflection time/depth, coherency, layer thickness, amplitude, roughness, etc.

Because only the low-frequency component is of interest, the prediction problem is isolated to a number of geologically defined horizons, such as, in this case, top chalk, base chalk, etc. At these horizons, a set of corresponding impedance measurements $(\mathbf{d})$ and seismic attributes $(\mathbf{m})$ are available. Through the learning process, the problem is narrowed to a function $\mathrm{G}$ that maps the seismic attributes into impedance:

$$
\mathbf{d}=\mathbf{G}(\mathbf{m})
$$

Once $\mathbf{G}$ has been found, the problem becomes trivial; to estimate $\mathbf{d}$ at all points where a set of attributes, $\mathbf{m}$, is available. $\mathrm{G}$ could be calculated using linear regression, resulting in a solution that optimally fits the data used. However, in general it does not lead to meaningful predictions because data are noisy. Instead, this equation is solved for G using a damped-weighted, least-squares algorithm. A weight is assigned to each attribute, linking the effect of each attribute to the estimated mapping operator G. A damping factor controls the amount of prediction variation. A small damping factor results in small prediction errors (i. e., high resolution) at known well sites but may also be very unstable away from the wells. Therefore, the damping factor controls the trade-off between high resolution (or high accuracy) and low stability versus low resolution and high stability.

The damping factor and the weights can be adjusted and fixed manually, but the quality of the estimated mapping operator is significantly enhanced by a Monte Carlo search to find the optimal values. The optimal values are chosen as those that produce the smallest cross-validation error. The cross-validation error is the mean of the error for all wells, calculated by removing each well in turn from the learning process and taking the difference between the estimated impedance and the true observed value.

The cross-validation approach ensures the estimated prediction operator performs equally well away from the known data as at the input well locations.

Uncertainty. Uncertainties are estimated for the mapping operator $\mathbf{G}$ and the final impedance measurement by propagating uncertainties of the input data through the learning and prediction process, respectively. A number of realizations of data and attributes with random noise added, are calculated. These sets of data are used to derive different estimates of $\mathbf{G}$, which will lead to uncertainty estimates of $\mathbf{G}$. Thus, uncertainty of the mapping operator depends only on the corresponding sets of well-log data and attributes measured at the well locations.

Uncertainty of the final estimated impedance is found by calculating a number of realizations of attributes with added random noise. From these attribute sets, a number of estimated impedance measurements is obtained. The variation of the estimated impedance will reflect the uncer- 


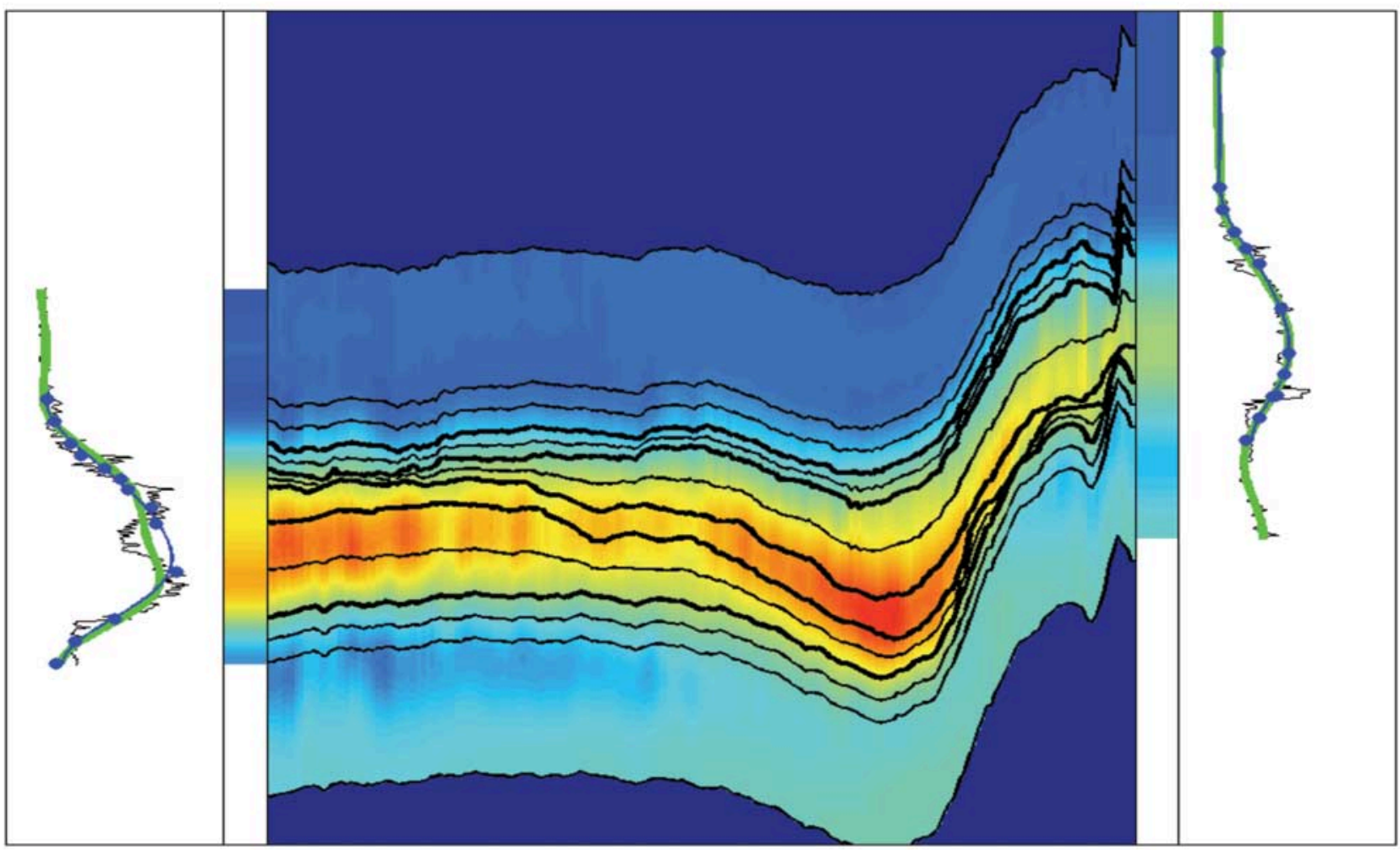

Figure 5. A profile between two wells showing interpolated horizons (thick black lines) and pseudohorizons (thin black lines) inserted to improve resolution. The original well log is shown as a black curve, the high-cut filtered log in green and as a color-density display and the predicted impedance as a blue curve. The predicted impedance along the profile between the two wells is shown as a color-density display with high impedance in yellow and red.

tainty of the impedance prediction, which can easily be calculated as the standard deviation of the estimated impedances.

Thus, the uncertainty measurements of the predicted impedance result from a combination of the uncertainty of the mapping operator (which is constant in the investigated area), and the propagated uncertainty of the attribute set used for prediction (which can vary locally in the investigated area).

In the examples shown in this article, eight realizations of input data provided stable uncertainty estimates.

The method applied to low-frequency impedance prediction. For a typical application, a 3D cube of seismic data, well data, and seismic horizons given as two-way traveltime maps are required. The corresponding attributes are derived from the seismic data. The technique presented above is applied to each of the interpreted horizons or geologic events. The individual steps of the simplified eventbased prediction are as follows:

(1) Parameterization: For each horizon, a corresponding set of attributes, $\mathbf{m}$, and impedance $\mathbf{d}$ is found, taken as the values where the low-pass-filtered impedance logs cross the event horizon.

(2) Learning: A combination of optimized cross validation and damped least squares allows $\mathbf{G}$ and the uncertainty of $\mathbf{G}$ to be determined at each event horizon.

(3) Prediction: Estimation of the low-frequency impedance along each event horizon is now as trivial as applying $\mathrm{G}$ to the attribute vectors in the area: $\mathbf{d}=\mathbf{G}(\mathbf{m})$. Uncertainty of $\mathbf{d}$ is obtained by propagating the uncertainties of the attributes, $\mathbf{m}$, and the uncertainty of $\mathbf{G}$, through the prediction formula.
(4) Deparameterization: Finally, a vertical 1D interpolation is applied everywhere in the cube, producing a full 3D representation of the low-frequency impedance and the associated estimation uncertainty.

Figure 5 shows a 2D profile between two wells in order to illustrate the steps described above. The original well logs are shown in black, and their high-cut filtered versions are shown in green. The predicted results are shown in blue. The seismic interpretation normally allows only a certain vertical resolution in the layer definition, in this case at 50-100 ms intervals, shown as thick black lines on Figure 5. Compared to the vertical and lateral variability in the seismic and log data, this may be an underrepresentation of the data information, and the option therefore exists to introduce a number of pseudohorizons, shown as thin black lines in Figure 5. Along these horizons, the input data points are derived from the high-cut filtered well logs. For each of the horizons, the learning process estimates the mapping function G, allowing the low-frequency impedance to be estimated at any point along each event horizon. Finally, onedimensional vertical interpolation is applied between event horizons, resulting in the predicted 3D impedance cube. The predicted impedance along a 2D profile is displayed in Figure 5 in color. Using one of the two wells shown in the profile as a blind well, this kind of display provides a useful QC tool by comparing predicted and measured impedance values. Note that it is important to utilize the surfaces in order to capture the inflection points, even on low-frequency logs, as accurately as possible. In Figure 5, some points on the upper half of the log (left) could have been omitted, but in the lower part of the chalk, omission of any one of the prediction points would significantly change the 


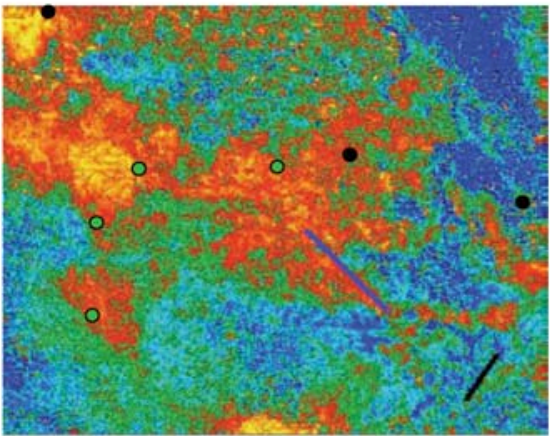

Impedance map
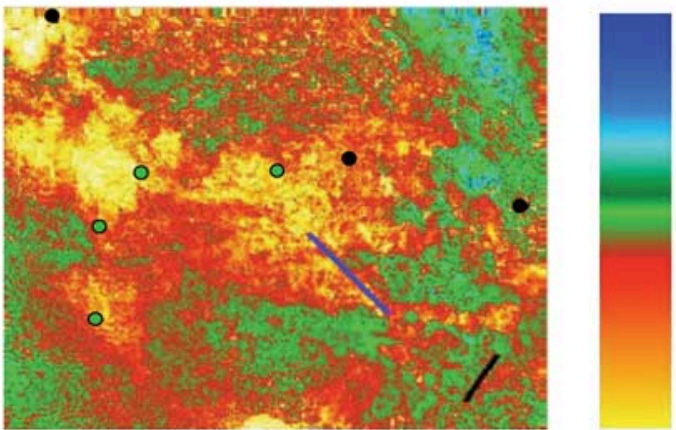

Low

Porosity map

Figure 6. 1998 results: Impedance map (upper) and porosity map (lower) for the zone of interest. Only the wells in black have been used to derive these 1998 results (as well as a number of wells outside of this area). Wells in green and purple were drilled after these results had been derived.

resulting log. This situation can be reversed as the layer thickness varies laterally, and, therefore, oversampling at one location may be necessary in order to allow adequate sampling at another location. At this location, it is the upper chalk that is oversampled and the lower chalk that is adequately sampled, but away from this location it may become the other way around.

Examples from the Danish North Sea. In 1998, a comprehensive study was carried out on the chalk in the Central Graben area of the Danish North Sea with the objective of providing a detailed model of porosity variations in the area. Seismic inversion was a significant part of this project, and special attention was paid to proper modeling of the low-frequency component.

During that project, seismic velocity and thickness of interpreted chalk units were found to be the two attributes with most significant influence on low-frequency impedance distribution. Seismic velocity was used as an important parameter with direct influence on impedance because welllog impedance is derived from velocity logs, and because it contains some information about compaction distributed laterally. Pelagic deposition of chalk is the dominant sedimentary process in the area, and under the normal compaction trend, it can be expected that the variation of the general increase of impedance with depth would be well captured by the seismic velocity. The unit thickness was used as it contains geologic information, such as sedimentation, compaction, and subsidence rate. The distance to the wells was also included in order to control the influence of distant wells with similar interval velocity and unit thickness. These attributes were combined to generate weights at each
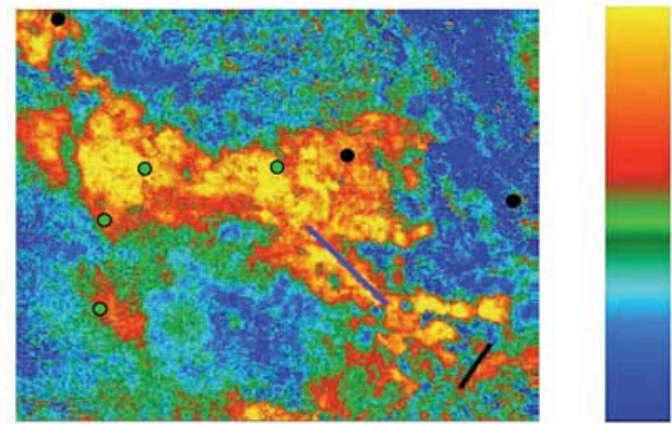

Low

Impedance map
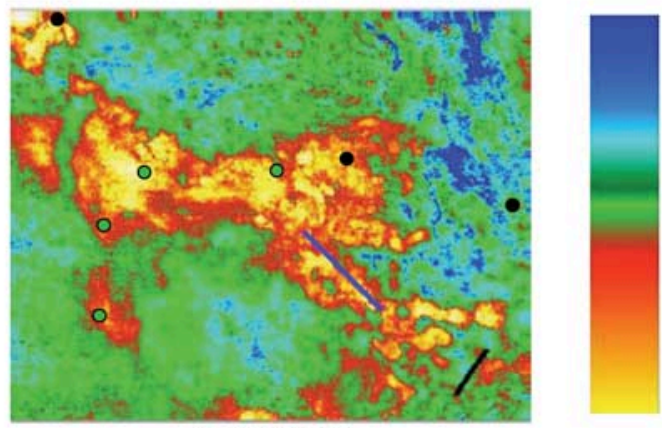

Low

High

High

Figure 7. Present results: impedance map (upper) and porosity map (lower) for the zone of interest. Wells displayed in green and in black were used for this prediction, and the deviated well displayed in purple was drilled based on these results.

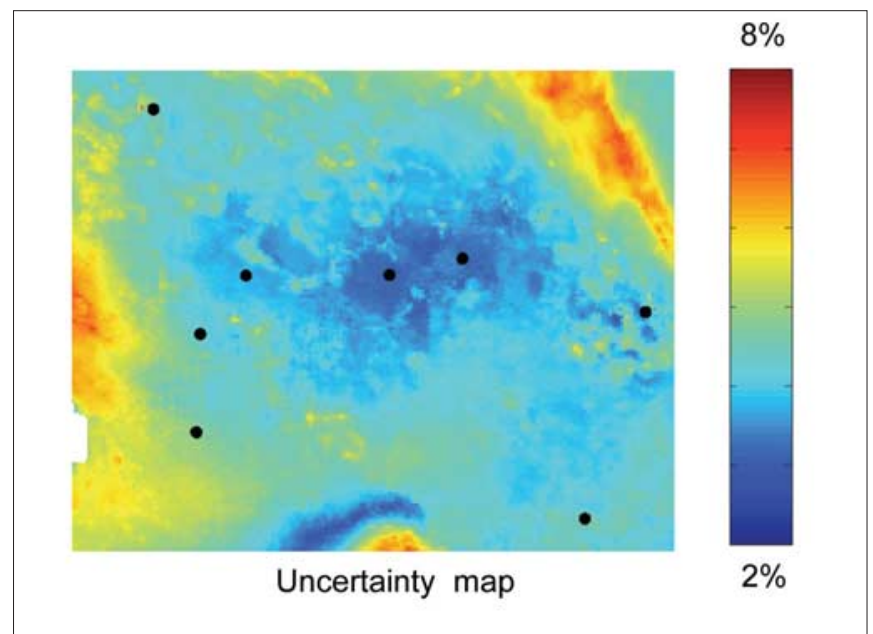

Figure 8. Absolute impedance uncertainty along interpreted top chalk horizon.

well location, which were then used to guide the interpolation for the whole project. In this way, a simple distancebased weight was converted into a more complex attribute containing important geologic information.

Careful preprocessing and velocity analysis were essential elements in deriving reliable seismic velocity information. Rather than using conventional stacking velocities, horizon-consistent velocity analysis (HVA) was performed and so in order to ensure high lateral and vertical resolution that the high semblance picks correspond to relevant 


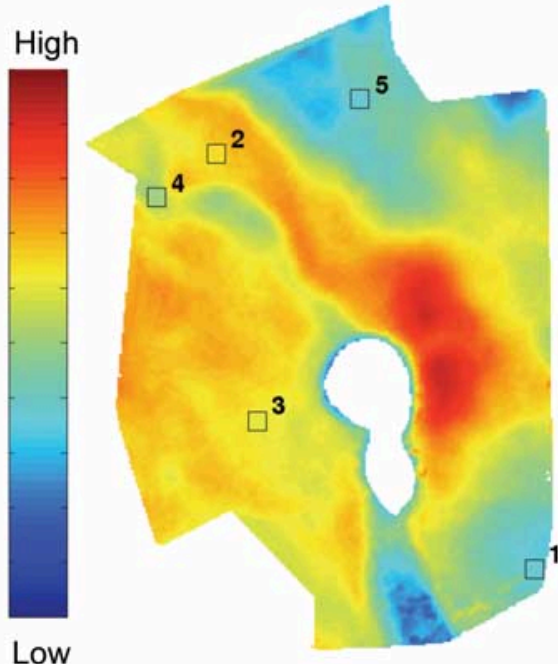

Impedance map

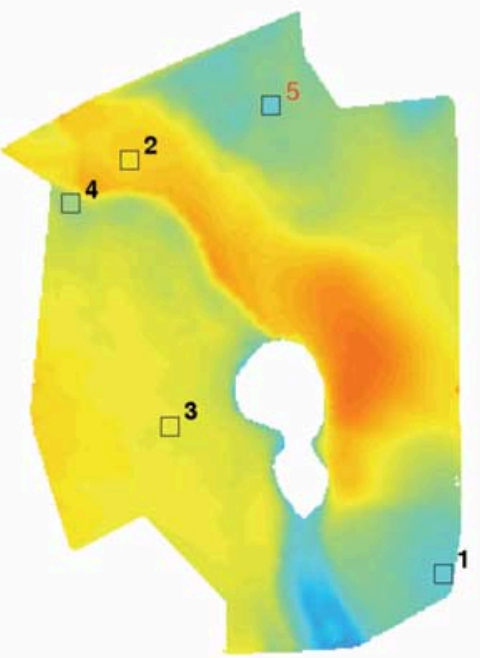

Impedance map
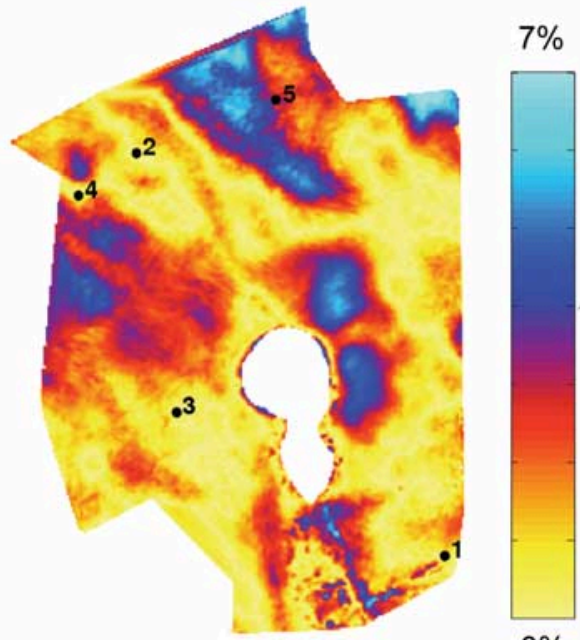

Relative difference

Figure 9. Comparison of the prediction of low-frequency impedance when all five wells were used (left) and when well 5 was excluded (middle). Relative difference between the two in percent shown on the right.

geologic layers. Velocities picked along the top and base of chalk were used to calculate interval velocity of the chalk layer using the Dix formula.

An average impedance map within the high-porosity upper chalk layer, resulting from the 1998 project, is shown in the upper part of Figure 6. This article focuses only on a small subset of the total area. Although a large number of wells were used to build the low-frequency model, only the four wells displayed in black existed within this area at the time of the study; the wells shown in green and blue were drilled after 1998. The porosity of the subsequently drilled wells matched the predicted porosity very well. In most cases, average predicted porosity blocked at about $40 \mathrm{ft}(8$ $\mathrm{ms}$ ) matched the well velocity within \pm 2 porosity units.

The relationship used to calculate the porosity from the impedance was derived from a crossplot using a large well database similar to that shown in upper part of Figure 3. The derived porosity model has been extensively used in well planning and tested by numerous wells. The area represents an excellent testing ground for this newly developed method.

The objective of the present study was to update the chalk porosity model in a limited part of the original study area by including additional data from several wells drilled since 1998, and at the same time test the newly developed software. Only the low-frequency part (in this case, up to 8 $\mathrm{Hz}$ ) of the acoustic impedance was tested in the update. This revised low-frequency impedance model was subsequently merged with the existing band-limited impedance derived by the original seismic inversion volume.

The locations of four additional wells, available within the study area, are displayed in green in Figure 6. The input time horizons were updated and refined as the result of the initial impedance and porosity results. Horizon interpretations are a key component in guiding the calculation of the low-frequency model and in the selected attributes available in the data set. The interval velocity for the entire chalk layer used in 1998 was also available. Within the newly developed software, it was possible to test the relevance of different attributes and their correlation to the well-log response. In addition to the chalk interval velocity and time thickness of individual chalk units, the time horizons and seismic amplitudes extracted at the top chalk horizon showed a strong correlation to the impedance logs. The resulting optimized impedance and porosity maps are shown in Figure 7. Note the striking improvement of the areal definition of the high-porosity zone, compared to the 1998 results. This improvement is achieved largely from a better prediction of the low-frequency impedance, in particular in the range of $2-8 \mathrm{~Hz}$. The 1998 porosity prediction (Figure 6) already indicated the presence of the high-porosity area extending southeast, even though the four well logs were not available at the time. In the present study, an improved prediction of the areal extent of the high-porosity zone is possible, not only because of the availability of these log measurements, but more importantly, because of an optimized methodology for deriving the low-frequency acoustic impedance data. The extent of the high-porosity zone to the southeast has been confirmed by a recently drilled deviated well (displayed in purple).

Figure 8 shows the estimated absolute impedance uncertainty along the interpreted top chalk horizon. The display shows lower uncertainty along a NW-SE trend, which was not unexpected because the two corners in the NE and the SW areas have different geology than the main NW-SE trend. The prediction operator performs best along the NW-SE trend, since most wells sample the type of geology present along this trend, whereas areas with uncharacteristic attributes (possible changing geology), in the two corners with little well control, have maximum uncertainty.

To test the sensitivity of the method to the amount of well control, the low-frequency impedance was also modeled in an area where only a few wells were available. As in the previous example, a number of seismic attributes were available, including interval velocity of three chalk layers, traveltime horizons to these layers, thickness of these and overburden layers, and seismic amplitudes. Predicted low-frequency impedance along one of the chalk interfaces is shown in Figure 9. The color in the square boxes at the well locations corresponds to the true value measured in the wells. The display on the left shows the impedance resulting from a prediction when all five wells were used. The result in the middle shows the impedance predicted when well 5 was excluded from the calculation, and the map on 
the right shows the relative difference between the two predictions. The overall impedance distribution appears to be much smoother on the middle display, and the maximum difference between the two maps is less than $7 \%$. Nevertheless, the prediction with only four wells is still rather stable and the overall impedance trend is well captured. It should be noted that the effect of removing any of the other four wells is even less severe.

Conclusions. We presented a method to derive absolute impedance with enough accuracy to be used for porosity prediction in chalk. As shown on examples from the chalk in the Danish North Sea, the application of the method significantly enhanced the estimations of the absolute porosity. A more focused image of porosity anomalies shown in Figures 6 and 7 gives more confidence for well placement.

Event-based, low-frequency impedance modeling is an automated procedure used to predict low-frequency impedance data, making use of a range of seismic attributes. The tool is fast and practical, allowing us to browse through a large number of attributes to find those that have an impact on accurate porosity prediction. With this method, the interpolation of well impedance at any given location is driven by attributes and not by the distance to the well data. Using relatively few wells and a few attributes, it can produce reliable results of good quality. The quality of the prediction improves where reliable correlations to seismic attributes are possible, and, of course, with the number of well data points. The mapping operator, and the contribution of each attribute, can easily be validated. An uncertainty estimation is inherent in the method, and covers both uncertainty of the input data, and the "goodness" of the fit between the known well impedance and any given attribute. The availability of an estimate of the absolute uncertainty in the porosity estimate can be utilized in volumetric estimates of a specific reservoir. The algorithm assists in identification of regions where the geology is either insufficiently represented or different from that sampled by the wells. This information about geologic changes can further be exploited in a number of related reservoir characterization studies and help decide on the need and value of further appraisal.

Suggested reading. The Contiguous Area Chalk Study (Maersk Oil, 1988). "Use of multiattribute transforms to predict log properties from seismic data" by Hampson et al. (GEOPHYSICS, 2001). "Neural networks in reservoir characterization" by Herrera et al. (TLE, 2006). "Seismic-controlled nonlinear extrapolation of well parameters using neural networks" by Liu and Liu (GEOPHYSICS, 1998). "Using neural networks to predict porosity thickness from 3D seismic" by Trappe and Hellmich (First Break, 2000). "Attribute-guided well-log interpolation" by Hansen et al. (submitted to GEOPHYSICS, 2007). TLE

Acknowledgments: The research presented in this paper is the result of a research project between Maersk Oil and Gas, the Niels Bohr Institute, and Core A/S. Thomas Mejer Hansen wishes to acknowledge financial support from the Danish Research Council (SNF 5013-00-0021). The work described here would not have been possible without the valuable experience and contributions from a number of colleagues at Maersk Oil.

Corresponding author: tmh@gfy.ku.dk 